MIDPI MOL2NET, International Conference Series on Multidisciplinary Sciences

sciforum

\title{
A review of some methods that incorporate decision-maker preferences in multi-objective evolutionary optimization using a multi-criteria classification method
}

\author{
J. Patricia Sánchez-Solís ${ }^{*}$, Eduardo Fernández ${ }^{b}$, Laura Cruz-Reyes ${ }^{c}$, Gilberto Rivera- \\ Zarate $^{a}$, Luis Cisneros ${ }^{a}$ \\ ${ }^{a}$ Autonomous University of Ciudad Juárez, julia.sanchez@uacj.mx, gilberto.rivera@uacj.mx, \\ al183002@alumnos.uacj.mx \\ ${ }^{b}$ Autonomous University of Sinaloa, eddyf171051@gmail.com \\ ${ }^{c}$ Madero Institute of Technology, lauracruzreyes@itcm.edu.mx
}



\begin{abstract}
.
In the real world there are many problems which involve the optimization of multiple objective functions at the same time. These are known as Multi-objective Optimization Problems (MOPs). Solving this kind of problems implies generating a set of good solutions, commonly known as Pareto-optimal solutions. The Multi-Objective Evolutionary Algorithms (MOEAs) have been extensively used to address this type of problems, since it allowing to get a set of the Pareto solutions in a particular run. Nevertheless, finding this solution set does not resolve the problem since the Decision-Maker (DM) still must select from that set the solution that matches more with his/her preferences. Determine the Region of Interest (RoI), in accordance with the DM's preferences, is an option that would make easy the selection process. The RoI has been defined as the region on the Pareto frontier which suits better to the DM's preferences. In order to help the DM in the selection process, different approaches in literature have added preferential information into the optimization process to lead the search towards the RoI.
\end{abstract}


Such is the case of the approach presented by (Cruz-Reyes et al., 2017) called Hybrid MultiCriteria Sorting Genetic Algorithm (H-MCSGA). This method addresses the preferences incorporation a priori into a MOEA to characterize the RoI by a multicriteria sorting method called THESEUS (Fernandez et al., 2011). H-MCSGA consists by two phases. First, a metaheuristic is used to create a set of solutions (reference set) that are assigned to ordered classes by the DM. The objective of this process is that the DM's preferences are indirectly reflected in this set. In the second phase, THESEUS is incorporated into an evolutionary algorithm to sort the new solutions created during optimization process. For this, THESEUS uses the reference set, generating selective pressure in the direction of the RoI. The performance of H-MCSGA was verified using nine instances of a public project portfolio problem. The achieve results show that $H$ $M C S G A$ reach a good definition of the RoI and outperforms the well-known NSGA-II (Deb et al., 2002). A first interactive version of the $H$ MCSGA is presented in (Cruz-Reyes et al., 2014), where the reference set is updated, only once, while exploration process. Consequently, the DM's preferences are updated. In examples on the portfolio problem, this proposal maintains its superiority over the NSGAII.

Finally, an interactive method more robust is proposed in (Cruz-Reyes et al., 2016) called the Interactive Multi-Criteria Sorting Genetic Algorithm (I-MCSGA). This method allows the $D M$ to assimilate progressively respecting the problem and to clarify his/her preferences. IMCSGA was assessed on project portfolio optimization problems. This algorithm was measure against with NSGA-II in three and four objectives problems and in nine and sixteen objectives problems with A2-NSGA-III (Jain, Deb, 2013). I-MCSGA presented better outcomes than these algorithms in regard to Pareto- 
dominance and to its ability to accomplish the

RoI. The automatic-enhancement procedure results efficient to include new solutions into the reference set, aiding THESEUS to propose more suitable assignments. Moreover, the proposed procedure to update preferences interactively is efficient to validate the enhanced reference set, still when the real DM was supplanted by the preference model proposed by (Fernandez et al., 2011). Therefore, I-MCSGA shown its capacity to identify the RoI and, to address optimization problems with a few and many numbers of objectives, effectively.

Future research directions should be leads towards extend the experimentation to problems where the true Pareto frontier is known, the use of others multicriteria sorting methods and the comparing with others most recent MOEAs. This with the aim of validating the outcomes of these approach with greater certainty.

\section{References}

Cruz-Reyes, L., Fernandez, E., Sanchez, P., Coello, C. A. C., \& Gomez, C. (2017). Incorporation of implicit decision-maker preferences in multi-objective evolutionary optimization using a multi-criteria classification method. Applied Soft Computing, 50, 48-57.

E. Fernandez, J. Navarro, A new approach to multi-criteria sorting based on fuzzy outranking relations: the THESEUS method, Eur. J. Oper. Res. 213 (2011) 405-413.

Deb, K., Pratap, A., Agarwal, S., Meyarivan, T.: A fast and elitist multiobjective genetic algorithm: NSGA-II. IEEE T. Evol. Comput. 6(2), pp. 182-197 (2002).

Cruz-Reyes, L., Fernandez, E., Gomez, C., \& Sanchez, P. (2014). Preference incorporation into evolutionary multiobjective optimization using a multi-criteria evaluation method. In Recent Advances on Hybrid Approaches for Designing Intelligent Systems (pp. 533-542). Springer, Cham.

Cruz-Reyes, L., Fernández, E., Sánchez, P., \& Gómez, C. (2016). Incorporation of decision-maker preferences in an interactive evolutionary multi-objective algorithm using a multi-criteria sorting. International Journal of Combinatorial Optimization Problems and Informatics, 7(3), 28-43.

Jain, H., Deb, K.: An improved adaptive approach for elitist nondominated sorting genetic algorithm for many-objective optimization. In Evolutionary Multi-Criterion Optimization, Springer Berlin Heidelberg, pp. 307-321 (2013). 
Fernandez, E., Lopez, E., Lopez, F., Coello, C.A.: Increasing selective pressure towards the best compromise in evolutionary multiobjective optimization: The extended NOSGA method. Inform. Sciences, 181(1), pp. 44-56 (2011). 